PROCEEDINGS OF THE

AMERICAN MATHEMATICAL SOCIETY

Volume 137, Number 12, December 2009, Pages 4083-4088

S 0002-9939(09)10015-1

Article electronically published on July 30, 2009

\title{
A DIRECT PROOF OF THE THEOREM ON FORMAL FUNCTIONS
}

\author{
FERNANDO SANCHO DE SALAS AND PEDRO SANCHO DE SALAS
}

(Communicated by Ted Chinburg)

\begin{abstract}
We give a direct and elementary proof of the theorem on formal functions by studying the behaviour of the Godement resolution of a sheaf of modules under completion. This proof also works in some non-noetherian cases.
\end{abstract}

\section{INTRODUCTION}

Let $\pi: X \rightarrow \operatorname{Spec} A$ be a proper scheme over a noetherian ring $A$. Let $\mathcal{M}$ be a coherent $\mathcal{O}_{X}$-module and $Y \subset \operatorname{Spec} A$ a closed subscheme. Let us denote by ${ }^{\wedge}$ the completion along $Y$ (respectively, along $\pi^{-1}(Y)$ ). The theorem on formal functions states that

$$
H^{i}(X, \mathcal{M})^{\wedge}=H^{i}(X, \hat{\mathcal{M}}) .
$$

Two important corollaries of this theorem are Stein's factorization theorem and

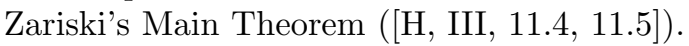

Hartshorne $[\mathrm{H}]$ gives a proof of the theorem on formal functions for projective schemes (over a noetherian ring). Grothendieck Gro proves it for proper noetherian schemes. He first gives sufficient conditions for the commutation of the cohomology of complexes of $A$-modules with inverse limits (Gro, 0, 13.2.3]); secondly, he gives a general theorem on the commutation of the cohomology of sheaves with inverse limits ([Gro, 0,13.3.1]); finally, he laboriously checks that the theorem on formal functions is under the hypothesis of this general one ([Gro, 4.1.5]).

In this paper we give the "obvious direct proof" of the theorem on formal functions. Very briefly, we prove that the completion of the Godement resolution of a coherent sheaf is a flasque resolution of the completion of the coherent sheaf and that taking sections in the Godement complex commutes with completion. This, together with the finiteness theorem for the higher direct images of a proper morphism, yields the theorem. In fact, we prove that in the non-noetherian case the theorem on formal functions still holds under some finiteness hypothesis.

Received by the editors July 2, 2008, and, in revised form, April 26, 2009.

2000 Mathematics Subject Classification. Primary 14A15; Secondary 14F99.

Key words and phrases. Formal functions, completion, cohomology.

The first author was supported by research projects MTM2006-04779 (MEC) and SA001A07 (JCYL).

(C)2009 American Mathematical Society Reverts to public domain 28 years from publication 


\section{Completion of Godement Resolution}

Definition 1. Let $X$ be a scheme, $\mathfrak{p} \subset \mathcal{O}_{X}$ a sheaf of ideals and $\mathcal{M}$ an $\mathcal{O}_{X}$-module. The $\mathfrak{p}$-adic completion of $\mathcal{M}$, denoted by $\widehat{\mathcal{M}}$, is

$$
\widehat{\mathcal{M}}:=\lim _{\leftarrow} \mathcal{M} / \mathfrak{p}^{n} \mathcal{M}
$$

If $U=\operatorname{Spec} A$ is an affine open subset and $I=\mathfrak{p}(U)$, one has a natural morphism

$$
\Gamma(U, \mathcal{M}) \otimes_{A} A / I^{n} \rightarrow \Gamma\left(U, \mathcal{M} / \mathfrak{p}^{n} \mathcal{M}\right)
$$

and then a morphism

$$
\Gamma(U, \mathcal{M})^{\wedge} \rightarrow \Gamma(U, \widehat{\mathcal{M}}),
$$

where $\Gamma(U, \mathcal{M})^{-}$is the $I$-adic completion of $\Gamma(U, \mathcal{M})$.

Definition 2. We say that $\mathcal{M}$ is affinely $\mathfrak{p}$-acyclic if for any affine open subset $U$ and any natural number $n$, the sheaves $\mathcal{M}$ and $\mathcal{M} / \mathfrak{p}^{n} \mathcal{M}$ are acyclic on $U$ and the morphism $\Gamma(U, \mathcal{M}) \otimes_{A} A / I^{n} \rightarrow \Gamma\left(U, \mathcal{M} / \mathfrak{p}^{n} \mathcal{M}\right)$ is an isomorphism. In particular, $\Gamma(U, \mathcal{M})^{\wedge} \rightarrow \Gamma(U, \widehat{\mathcal{M}})$ is an isomorphism.

Every quasi-coherent module is affinely $\mathfrak{p}$-acyclic (with $\mathfrak{p}$ quasi-coherent).

Notation. For any sheaf $F$, let us denote

$$
0 \rightarrow F \rightarrow C^{0} F \rightarrow C^{1} F \rightarrow \cdots \rightarrow C^{n} F \rightarrow \cdots
$$

as its Godement resolution (see [Go, II, 4.3]). We shall also denote $C^{\cdot} F=\bigoplus_{i \geq 0} C^{i} F$ and $F_{i}=\operatorname{Ker}\left(C^{i} F \rightarrow C^{i+1} F\right)$. One has that $C^{0} F_{i}=C^{i} F$. The functors $F \rightsquigarrow C^{i} F$ and $F \rightsquigarrow F_{i}$ are exact.

Lemma 3. Let $X$ be a scheme, $\mathfrak{p}$ a sheaf of ideals of finite type on $X$ and $\mathcal{M}$ an $\mathcal{O}_{X}$-module. Denote $I=\Gamma(X, \mathfrak{p})$ and assume that $\mathfrak{p}$ is generated by a finite number of global sections (this holds for example when $X$ is affine). For any open subset $V \subseteq X$ one has

$$
\Gamma\left(V, C^{0}(\mathfrak{p} \mathcal{M})\right)=I \cdot \Gamma\left(V, C^{0} \mathcal{M}\right) .
$$

In particular, the natural morphism $\mathfrak{p} C^{0} M \rightarrow C^{0}(\mathfrak{p} \mathcal{M})$ is an isomorphism.

Proof. If $J$ is a finitely generated ideal of a ring $A$ and $M_{i}$ is a collection of $A$ modules, then $J \cdot \prod M_{i}=\prod\left(J \cdot M_{i}\right)$. Now, by hypothesis, $\mathfrak{p}$ is generated by a finite number of global sections $f_{1}, \ldots, f_{r}$. Let $J=\left(f_{1}, \ldots, f_{r}\right)$. Then

$$
\Gamma\left(V, C^{0}(\mathfrak{p} \mathcal{M})\right)=\prod_{x \in V} \mathfrak{p}_{x} \cdot \mathcal{M}_{x}=\prod_{x \in V} J \cdot \mathcal{M}_{x}=J \cdot \prod_{x \in V} \mathcal{M}_{x}=J \cdot \Gamma\left(V, C^{0} \mathcal{M}\right) .
$$

Since $I \cdot \prod_{x \in V} \mathcal{M}_{x}$ is contained in $\Gamma\left(V, C^{0}(\mathfrak{p} \mathcal{M})\right)$ one concludes the lemma. In particular, if $V$ is affine, then $\Gamma\left(V, C^{0}(\mathfrak{p} \mathcal{M})\right)=I_{V} \cdot \Gamma\left(V, C^{0} \mathcal{M}\right)$, with $I_{V}=\Gamma(V, \mathfrak{p})$. It follows that $\mathfrak{p} C^{0} M \rightarrow C^{0}(\mathfrak{p} \mathcal{M})$ is an isomorphism.

Proposition 4. Let $X$ be a scheme and let $\mathfrak{p}$ be an ideal of finite type. For any $\mathcal{O}_{X}$-module $\mathcal{M}$ one has:

(1) $\mathfrak{p} C^{i} \mathcal{M}=C^{i}(\mathfrak{p} \mathcal{M})$ and $\left(C^{i} \mathcal{M}\right) / \mathfrak{p}\left(C^{i} \mathcal{M}\right)=C^{i}(\mathcal{M} / \mathfrak{p} \mathcal{M})$, for any $i$.

(2) $\mathfrak{p} \mathcal{M}_{i}=(\mathfrak{p} \mathcal{M})_{i}$ and $(\mathcal{M} / \mathfrak{p} \mathcal{M})_{i}=\mathcal{M}_{i} / \mathfrak{p} \mathcal{M}_{i}$, for any $i$.

(3) $C^{i} \mathcal{M}$ is affinely $\mathfrak{p}$-acyclic. 
(4) $\widehat{C^{i} \mathcal{M}}$ is flasque. Moreover, if $\mathfrak{p}$ is generated by a finite number of global sections, then

$$
\Gamma\left(X, \widehat{C^{i \mathcal{M}}}\right)=\Gamma\left(X, C^{i} \mathcal{M}\right)^{\wedge} .
$$

Proof. (1) and (2). We may assume that $X$ is affine. Hence $\mathfrak{p} C^{0} \mathcal{M}=C^{0}(\mathfrak{p} \mathcal{M})$ by the previous lemma and $\left(C^{0} \mathcal{M}\right) / \mathfrak{p} C^{0} \mathcal{M}=C^{0} \mathcal{M} / C^{0}(\mathfrak{p} \mathcal{M})=C^{0}(\mathcal{M} / \mathfrak{p} \mathcal{M})$. From the exact sequence

$$
\mathcal{M} / \mathfrak{p} \mathcal{M} \rightarrow C^{0} \mathcal{M} / \mathfrak{p} C^{0} \mathcal{M} \rightarrow \mathcal{M}_{1} / \mathfrak{p} \mathcal{M}_{1} \rightarrow 0
$$

and the isomorphism $C^{0} \mathcal{M} / \mathfrak{p} C^{0} \mathcal{M}=C^{0}(\mathcal{M} / \mathfrak{p} \mathcal{M})$, it follows that $\mathcal{M}_{1} / \mathfrak{p} \mathcal{M}_{1}=$ $(\mathcal{M} / \mathfrak{p} \mathcal{M})_{1}$. By the exactness of the functor $M \rightsquigarrow M_{1}$ one concludes that $\mathfrak{p} \mathcal{M}_{1}=$ $(\mathfrak{p} \mathcal{M})_{1}$. Consequently $\mathfrak{p} C^{1} \mathcal{M}=\mathfrak{p} C^{0}\left(\mathcal{M}_{1}\right)=C^{0}\left(\mathfrak{p} \mathcal{M}_{1}\right)=C^{0}\left((\mathfrak{p} \mathcal{M})_{1}\right)=C^{1}(\mathfrak{p} \mathcal{M})$, and analogously $C^{1} \mathcal{M} / \mathfrak{p} C^{1} \mathcal{M}=C^{1}(\mathcal{M} / \mathfrak{p} \mathcal{M})$. Repeating this argument one concludes (1) and (2).

3. Since $C^{i} \mathcal{M}=C^{0} \mathcal{M}_{i}$, we may assume that $i=0$. Denote $\mathcal{N}=C^{0} \mathcal{M}$. By (1), $\mathcal{N} / \mathfrak{p}^{n} \mathcal{N}$ is acyclic on any open subset. From the long exact sequence of cohomology associated to $0 \rightarrow \mathfrak{p}^{n} \mathcal{N} \rightarrow \mathcal{N} \rightarrow \mathcal{N} / \mathfrak{p}^{n} \mathcal{N} \rightarrow 0$ and the acyclicity of $\mathfrak{p}^{n} \mathcal{N}$ (by (1)), one obtains that

$$
\Gamma\left(U, \mathcal{N} / \mathfrak{p}^{n} \mathcal{N}\right)=\Gamma(U, \mathcal{N}) / \Gamma\left(U, \mathfrak{p}^{n} \mathcal{N}\right)
$$

Moreover, if $U$ is affine, $\Gamma\left(U, \mathfrak{p}^{n} \mathcal{N}\right)=\mathfrak{p}^{n}(U) \Gamma(U, \mathcal{N})$, by Lemma 3 . We have concluded (3).

(4) Again, we may assume that $i=0$. Let us prove that the completion of $\mathcal{N}=C^{0} \mathcal{M}$ is flasque. It suffices to prove that its restriction to any affine open subset is flasque, so we may assume that $X$ is affine. Let us denote $I=\mathfrak{p}(X)$. For any open subset $V$, one has as in the proof of (3)

$$
\Gamma(V, \widehat{\mathcal{N}})=\lim _{\leftarrow} \Gamma\left(V, \mathcal{N} / \mathfrak{p}^{n} \mathcal{N}\right)=\lim _{n} \Gamma(V, \mathcal{N}) / \Gamma\left(V, \mathfrak{p}^{n} \mathcal{N}\right)
$$

and by Lemma 3, $\Gamma\left(V, \mathfrak{p}^{n} \mathcal{N}\right)=I^{n} \Gamma(V, \mathcal{N})$. In conclusion, $\Gamma(V, \widehat{\mathcal{N}})=\Gamma(V, \mathcal{N})^{-}$. One concludes that $\widehat{\mathcal{N}}$ is flasque because $\mathcal{N}$ is flasque and that the $I$-adic completion preserves surjections. The same arguments prove the second part of the statement.

Lemma 3 and Proposition 4 will be used very often in the sequel without referencing.

Proposition 5. Let $\mathfrak{p}$ be of finite type and $\mathcal{M}$ be affinely $\mathfrak{p}$-acyclic. Then $\widehat{C \cdot \mathcal{M}}$ is a flasque resolution of $\widehat{\mathcal{M}}$.

Proof. We already know that $\widehat{C \cdot \mathcal{M}}$ is flasque. Let us prove now that $\mathcal{M}_{1}$ is affinely $\mathfrak{p}$-acyclic. From the exact sequence

$$
0 \rightarrow \mathcal{M} / \mathfrak{p}^{n} \mathcal{M} \rightarrow C^{0}\left(\mathcal{M} / \mathfrak{p}^{n} \mathcal{M}\right) \rightarrow \mathcal{M}_{1} / \mathfrak{p}^{n} \mathcal{M}_{1} \rightarrow 0
$$

one has that $\mathcal{M}_{1} / \mathfrak{p}^{n} \mathcal{M}_{1}$ is acyclic on any affine open subset. Moreover, taking sections on an affine open subset $U=\operatorname{Spec} A$, one obtains the exact sequence (let us denote $I=\mathfrak{p}(U))$

$$
0 \rightarrow \Gamma(U, \mathcal{M}) \otimes_{A} A / I^{n} \rightarrow \Gamma\left(U, C^{0} \mathcal{M}\right) \otimes_{A} A / I^{n} \rightarrow \Gamma\left(U, \mathcal{M}_{1} / \mathfrak{p}^{n} \mathcal{M}_{1}\right) \rightarrow 0
$$

and then $\Gamma\left(U, \mathcal{M}_{1}\right) \otimes_{A} A / I^{n}=\Gamma\left(U, \mathcal{M}_{1} / \mathfrak{p}^{n} \mathcal{M}_{1}\right)$; i.e. $\mathcal{M}_{1}$ is affinely $\mathfrak{p}$-acyclic. 
Now, taking the inverse limit in the above exact sequence (and taking into account that the $I$-adic completion preserves surjections) one obtains the exact sequence

$$
0 \rightarrow \Gamma(U, \widehat{\mathcal{M}}) \rightarrow \Gamma\left(U, \widehat{C^{0} \mathcal{M}}\right) \rightarrow \Gamma\left(U, \widehat{\mathcal{M}_{1}}\right) \rightarrow 0 .
$$

Therefore the sequence $0 \rightarrow \widehat{\mathcal{M}} \rightarrow \widehat{C^{0} \mathcal{M}} \rightarrow \widehat{\mathcal{M}_{1}} \rightarrow 0$ is exact. The conclusion follows easily.

Remark 6 . From the exact sequence $(*)$ one obtains that $H^{1}(U, \widehat{\mathcal{M}})=0$ for any affine open subset $U$ and any affinely $\mathfrak{p}$-acyclic module $\mathcal{M}$. Since $\mathcal{M}_{1}$ is also affinely $\mathfrak{p}$-acyclic, one has $H^{2}(U, \widehat{M})=0$. Recurrently, $H^{i}(U, \widehat{\mathcal{M}})=0$ for any $i>0$. Moreover, $\widehat{\mathcal{M}} / \mathfrak{p}^{n} \widehat{\mathcal{M}}=\mathcal{M} / \mathfrak{p}^{n} \mathcal{M}$. In fact, $\widehat{\mathcal{M}} / \mathfrak{p}^{n} \widehat{\mathcal{M}}$ is the sheaf associated to the presheaf $\Gamma(U, \widehat{\mathcal{M}}) / I^{n} \Gamma(U, \widehat{\mathcal{M}})$, with $I=\mathfrak{p}(U)$. Since $\Gamma(U, \widehat{\mathcal{M}})=\Gamma(U, \mathcal{M})^{\wedge}$, this presheaf is $\Gamma(U, \mathcal{M})^{\wedge} / I^{n} \Gamma(U, \mathcal{M})^{\wedge}=\Gamma(U, \mathcal{M}) / I^{n} \Gamma(U, \mathcal{M})$ (because $I$ is finitely generated), whose associated sheaf is $\mathcal{M} / \mathfrak{p}^{n} \mathcal{M}$. Finally

$$
\begin{aligned}
\Gamma\left(U, \mathcal{M} / \mathfrak{p}^{n} \mathcal{M}\right)=\Gamma(U, \mathcal{M}) / I^{n} \Gamma(U, \mathcal{M}) & =\Gamma(U, \mathcal{M})^{\wedge} / I^{n} \Gamma(U, \mathcal{M})^{\wedge} \\
& =\Gamma(U, \widehat{\mathcal{M}}) / I^{n} \Gamma(U, \widehat{\mathcal{M}}) .
\end{aligned}
$$

In conclusion, if $\mathfrak{p}$ is a finite type ideal and $\mathcal{M}$ is affinely $\mathfrak{p}$-acyclic, then $\widehat{\mathcal{M}}$ is also affinely $\mathfrak{p}$-acyclic. This result will not be used in the sequel.

\section{Finiteness hypothesis}

Definition 7. An $\mathcal{O}_{X}$-module $\mathcal{M}$ is said to be pseudo-coherent if for each $n \in \mathbb{N}$ there exists locally on $X$ an exact sequence $L_{n} \rightarrow \cdots \rightarrow L_{0} \rightarrow \mathcal{M} \rightarrow 0$, where $L_{i}$ are free modules of finite type. A morphism $f: X \rightarrow Y$ is said to be pseudo-coherent if every $x \in X$ has an open neighborhood $U$ such that the restriction $f_{\mid U}$ factors as $U \stackrel{i}{\rightarrow} Z \stackrel{p}{\rightarrow} Y$, where $i$ is a closed immersion such that $i_{*} \mathcal{O}_{U}$ is pseudo-coherent on $Z$, and $p$ is smooth (II, p. 228, Def. 1.2]).

Let $f: X \rightarrow Y$ be a pseudo-coherent morphism of schemes, $\mathcal{M}$ a pseudo-coherent $\mathcal{O}_{X}$-module and $\mathfrak{p}$ a sheaf of ideals on $Y$ of finite type. Let us denote $D_{\mathfrak{p}} \mathcal{O}_{Y}=$ $\bigoplus_{n \in \mathbb{N}} \mathfrak{p}^{n}$, which has an obvious structure of sheaf of rings. Moreover, $\bigoplus_{n \in \mathbb{N}} R^{i} f_{*}\left(\mathfrak{p}^{n} \mathcal{M}\right)$ has a natural structure of $D_{\mathfrak{p}} \mathcal{O}_{Y}$-module. We shall assume that

$$
\bigoplus_{n \in \mathbb{N}} R^{i} f_{*}\left(\mathfrak{p}^{n} \mathcal{M}\right) \text { is a } D_{\mathfrak{p}} \mathcal{O}_{Y} \text {-module of finite type. }
$$

Examples 8. Let $f: X \rightarrow Y, \mathcal{M}$ and $\mathfrak{p}$ be as above. Let us see some cases where the hypothesis (11) holds:

(a) Noetherian case: Assume that $f: X \rightarrow Y$ is a proper morphism between noetherian schemes. Then $f$ is pseudo-coherent, $\mathcal{M}$ is coherent and $\mathfrak{p}$ is a coherent sheaf of ideals. By Gro, 3.3.2], (11) holds. In fact, this statement is a consequence of the Finiteness Theorem: let $Y^{\prime}=\operatorname{Spec} D_{\mathfrak{p}} \mathcal{O}_{Y}, X^{\prime}=\operatorname{Spec} D_{\mathfrak{p}} \mathcal{O}_{X}$, and $\mathcal{M}^{\prime}=D_{\mathfrak{p}} \mathcal{M}=$ $\bigoplus_{n=0}^{\infty} \mathfrak{p}^{n} \mathcal{M}$, with the obvious $\mathcal{O}_{X^{\prime}}$-module structure and $f^{\prime}: X^{\prime} \rightarrow Y^{\prime}$ the natural morphism. $\mathcal{M}^{\prime}$ is a coherent $\mathcal{O}_{X^{\prime}}$-module and $f^{\prime}$ is proper. By the Finiteness Theorem $R^{i} f_{*}^{\prime} \mathcal{M}^{\prime}$ is a coherent $\mathcal{O}_{Y^{\prime}}$-module. Since $\pi: Y^{\prime} \rightarrow Y$ and $\pi^{\prime}: X^{\prime} \rightarrow X$ are affine morphisms, one concludes that $\pi_{*}\left(R^{i} f_{*}^{\prime} \mathcal{M}^{\prime}\right)=R^{i} f_{*}\left(\pi_{*}^{\prime} \mathcal{M}^{\prime}\right)=\bigoplus_{n \in \mathbb{N}} R^{i} f_{*}\left(\mathfrak{p}^{n} \mathcal{M}\right)$ is a $\pi_{*} \mathcal{O}_{Y^{\prime}}=D_{\mathfrak{p}} \mathcal{O}_{Y}$-module of finite type. 
(b) Assume that $f$ is proper and flat and that $\mathcal{M}$ is flat over $Y$. With the same notation as in the preceding example, one has that $f^{\prime}$ is a proper pseudo-coherent map (see [I, p. 233, Cor 1.10]) and $\mathcal{M}^{\prime} \simeq \mathcal{O}_{Y^{\prime}} \otimes_{\mathcal{O}_{Y}} \mathcal{M}$ is a pseudo-coherent module on $X^{\prime}$ (see [I, p. 111, Cor 2.16.1]). By Kiehl's Finiteness Theorem ([K, p. 315, Thm. 2.9']) $R^{i} f_{*}^{\prime} \mathcal{M}^{\prime}$ is a pseudo-coherent $\mathcal{O}_{Y^{\prime}}$-module. One concludes as before that (11) holds.

Theorem 9 (on formal functions). Let $f: X \rightarrow Y$ be a morphism of schemes, $\mathfrak{p}$ a sheaf of ideals of finite type on $Y$ and $\mathfrak{p} \mathcal{O}_{X}$ the ideal induced in $X$. Let $\mathcal{M}$ be an affinely $\mathfrak{p}$-acyclic $\mathcal{O}_{X}$-module satisfying (11). The natural morphisms (where completions are made by $\mathfrak{p}$ and $\mathfrak{p} \mathcal{O}_{X}$ respectively)

$$
\widehat{R^{i} f_{*} \mathcal{M}} \rightarrow R^{i} f_{*}(\widehat{\mathcal{M}})
$$

are isomorphisms. If $Y=\operatorname{Spec} A$, then

$$
H^{i}(X, \mathcal{M})^{\wedge}=H^{i}(X, \widehat{\mathcal{M}}) .
$$

Proof. The question is local on $Y$, so we may assume that $Y=\operatorname{Spec} A$ is affine. It

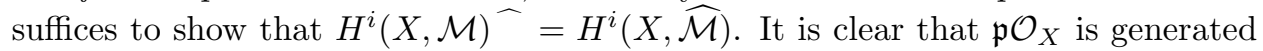
by the global sections $I=\Gamma(Y, \mathfrak{p})$.

Let $C \cdot \mathcal{M}$ be the Godement resolution of $\mathcal{M}$. Let us denote by $d_{i}$ the differential of the complex $\Gamma(X, C \cdot \mathcal{M})$ on degree $i$ and $d_{i}^{n}$ the differential of the complex $\Gamma\left(X, \mathfrak{p}^{n} C \cdot \mathcal{M}\right)=I^{n} \Gamma(X, C \cdot \mathcal{M})$ on degree $i$. Observe that $\operatorname{Im} d_{i}^{n}=I^{n} \operatorname{Im} d_{i}$ and $\operatorname{Ker} d_{i}=\Gamma\left(X, \mathcal{M}_{i}\right)$ because $\mathcal{M}_{i}$ is the kernel of $C^{i} \mathcal{M} \rightarrow C^{i+1} \mathcal{M}$ (recall that $\left.C^{i} \mathcal{M}=C^{0} \mathcal{M}_{i}\right)$. Moreover, $\left(I^{n} \operatorname{Ker} d_{i}\right) \cap \operatorname{Im} d_{i-1}=I^{n} \operatorname{Im} d_{i-1}$, as follows from the inclusion $I^{n} \operatorname{Im} d_{i-1} \subseteq\left(I^{n} \operatorname{Ker} d_{i}\right) \cap \operatorname{Im} d_{i-1}$ and the diagram

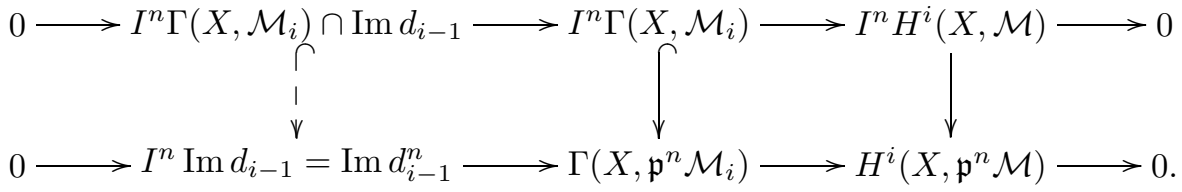

Hence,

$$
H^{i}(X, \mathcal{M})^{\wedge}=\left(\operatorname{Ker} d_{i} / \operatorname{Im} d_{i-1}\right)^{\wedge}=\widehat{\operatorname{Ker} d_{i}} / \widehat{\operatorname{Im} d_{i-1}}
$$

$\widehat{C \cdot M}$ is a flasque resolution of $\widehat{\mathcal{M}}$ (by Proposition 5 ) and $\Gamma(X, \widehat{C \cdot \mathcal{M}})=$ $\Gamma(X, C \cdot \mathcal{M})$ (by Proposition $4(4))$. Completing the exact sequences

$$
0 \rightarrow \operatorname{Ker} d_{i} \rightarrow \Gamma\left(X, C^{i} \mathcal{M}\right) \rightarrow \operatorname{Im} d_{i} \rightarrow 0
$$

we obtain the exact sequences

$$
0 \rightarrow \widehat{\operatorname{Ker} d_{i}} \rightarrow \Gamma\left(X, \widehat{C^{i \mathcal{M}}}\right) \rightarrow \widehat{\operatorname{Im} d_{i}} \rightarrow 0
$$

because, as we shall see below, the $I$-adic topology of $\Gamma\left(X, C^{i} \mathcal{M}\right)$ induces the $I$-adic topology on $\operatorname{Ker} d_{i}$. Then $H^{i}(\Gamma(X, \widehat{C \cdot \mathcal{M}}))=\widehat{\operatorname{Ker} d_{i}} / \widehat{\operatorname{Im} d_{i-1}}$ and the natural map $H^{i}(X, \mathcal{M})^{\wedge}=\left[H^{i} \Gamma\left(X, C^{\cdot \mathcal{M}}\right)\right]^{\wedge} \rightarrow H^{i}\left(\Gamma\left(X, C^{\cdot \mathcal{M}}\right)^{\wedge}\right)=H^{i}(\Gamma(X, \widehat{C \cdot \mathcal{M}}))=H^{i}(X, \widehat{\mathcal{M}})$ is an isomorphism.

Let us prove that the $I$-adic topology of $\Gamma\left(X, C^{i} \mathcal{M}\right)$ induces the $I$-adic topology on Ker $d_{i}=\Gamma\left(X, \mathcal{M}_{i}\right)$. Intersecting the equality $I^{n} \Gamma\left(X, C^{0} \mathcal{M}_{i}\right)=\Gamma\left(X, C^{0}\left(\mathfrak{p}^{n} \mathcal{M}_{i}\right)\right)$ with $\Gamma\left(X, \mathcal{M}_{i}\right)$, one obtains that the induced topology on $\Gamma\left(X, \mathcal{M}_{i}\right)$ is given by the filtration $\left\{\Gamma\left(X, \mathfrak{p}^{n} \mathcal{M}_{i}\right)\right\}$. Hence it suffices to show that this filtration is $I$-stable. 
Since $\mathfrak{p}^{n} \mathcal{M}_{i}=\left(\mathfrak{p}^{n} \mathcal{M}\right)_{i}$ (see Proposition $4(2)$ ), it is enough to prove that the filtration $\left\{\Gamma\left(X,\left(\mathfrak{p}^{n} \mathcal{M}\right)_{i}\right)\right\}$ is $I$-stable. This is equivalent to showing that $\bigoplus_{n=0}^{\infty} \Gamma\left(X,\left(\mathfrak{p}^{n} \mathcal{M}\right)_{i}\right)$ is a $D_{I} A$-module generated by a finite number of homogeneous components, where $D_{I} A=\bigoplus_{n=0}^{\infty} I^{n}$. By the exact sequence

$$
\bigoplus_{n=0}^{\infty} \Gamma\left(X, C^{i-1}\left(\mathfrak{p}^{n} \mathcal{M}\right)\right) \rightarrow \bigoplus_{n=0}^{\infty} \Gamma\left(X,\left(\mathfrak{p}^{n} \mathcal{M}\right)_{i}\right) \rightarrow \bigoplus_{n=0}^{\infty} H^{i}\left(X, \mathfrak{p}^{n} \mathcal{M}\right) \rightarrow 0
$$

it suffices to see the statement for the first and third members. The statement for the first one is obvious because $\Gamma\left(X, C^{i-1}\left(\mathfrak{p}^{n} \mathcal{M}\right)\right)=I^{n} \Gamma\left(X, C^{i-1} \mathcal{M}\right)$. For the third one, it is true by hypothesis (1).

Remark 10. Reading the above proof carefully, it is not difficult to see that one has already showed that $H^{i}(X, \mathcal{M})^{\wedge}=\lim _{n} H^{i}\left(X, \mathcal{M} / \mathfrak{p}^{n} \mathcal{M}\right)$

Corollary 11. Assume now that $f: X \rightarrow Y$ is a pseudo-coherent projective morphism of schemes; that is, locally on $Y, f$ factors as $X \stackrel{i}{\rightarrow} \mathbb{P}_{Y}^{r} \stackrel{\pi}{\rightarrow} Y$, where $i$ is a pseudo-coherent closed immersion and $\pi$ is the natural projection. Let $\mathcal{M}$ be a pseudo-coherent $\mathcal{O}_{X}$-module, flat over $Y$, and $\mathfrak{p}$ a sheaf of ideals on $Y$ of finite type. Then the theorem on formal functions holds:

$$
\widehat{R^{i} f_{*} \mathcal{M}} \stackrel{\sim}{\rightarrow} R^{i} f_{*}(\widehat{\mathcal{M}}) .
$$

Proof. The question is local on $Y$, so we can assume that $f$ factors as $X \stackrel{i}{\rightarrow} \mathbb{P}_{Y}^{r} \stackrel{\pi}{\rightarrow} Y$. The theorem holds obviously for $i$, i.e., $\widehat{i_{*} \mathcal{M}}=i_{*} \widehat{\mathcal{M}}$, and it holds also for $\pi$ (by Theorem 9). Then

$$
\left.\widehat{R^{i} f_{*} \mathcal{M}}=R^{i} \widehat{\pi_{*}\left(i_{*} \mathcal{M}\right.}\right)=R^{i} \pi_{*}\left(\widehat{i_{*} \mathcal{M}}\right)=R^{i} \pi_{*}\left(i_{*} \widehat{\mathcal{M}}\right)=R^{i} f_{*}(\widehat{\mathcal{M}}) .
$$

\section{REFERENCES}

[A] Atiyah, M.F. and Macdonald, I.G., Introduction to Commutative Algebra, Addison-Wesley Publishing Company, 1969. MR0242802 (39:4129)

[Go] Godement, R., Topologie algébrique et théorie des faisceaux, Troisième édition revue et corrigée. Publications de l'Institut de Mathématique de l'Université de Strasbourg, XIII. Actualités Scientifiques et Industrielles, No. 1252. Hermann, Paris, 1973. MR0345092 (49:9831)

[Gro] Grothendieck, A. and Dieudonné, J., Éléments de géométrie algébrique III, Publ. Math. IHES, 1961. MR0217085 (36:177c)

[H] Hartshorne, R., Algebraic Geometry, Grad. Texts Math., 52, Springer-Verlag, 1977. MR0463157 (57:3116)

[I] Illusie, L., Théorie des Intersections et Théorème de Riemann-Roch, Lecture Notes in Math., 225, Springer-Verlag, New York, 1971. MR0354655 (50:7133)

[K] Kiehl, R., Ein "Descente"-Lemma und Grothendiecks Projektionsatz für nicht-noetheresche Schemata, Math Annalen 198 (1972), 287-316. MR0382280(52:3165)

Departamento de Matemáticas, Universidad de Salamanca, Plaza de la Merced 1-4, 37008 Salamanca, Spain

E-mail address: fsancho@usal.es

Departamento de Matemáticas, Universidad de Extremadura, Avenida de Elvas s/n, 06071 BAdaJoz, Spain

E-mail address: sancho@unex.es 